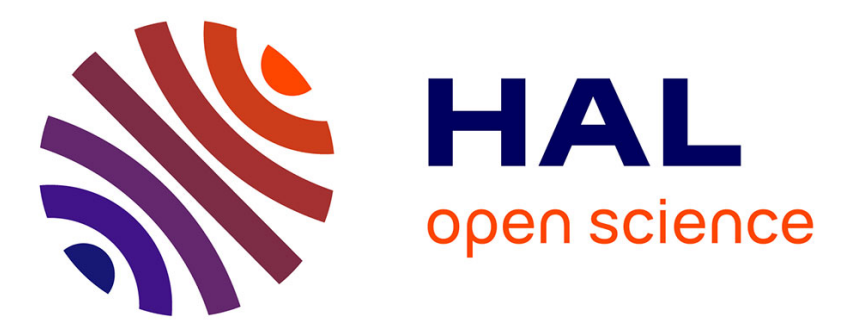

\title{
Optical properties of GaAs/AlGaAs quantum dots realized by implantation induced intermixing
}

\author{
F. Prins, F. Adler, G. Lehr, S. Nikitin, H. Schweizer, G. Smith
}

\section{To cite this version:}

F. Prins, F. Adler, G. Lehr, S. Nikitin, H. Schweizer, et al.. Optical properties of GaAs/AlGaAs quantum dots realized by implantation induced intermixing. Journal de Physique IV Proceedings, 1993, 03 (C5), pp.C5-115-C5-118. 10.1051/jp4:1993520 . jpa-00251606

\section{HAL Id: jpa-00251606 https://hal.science/jpa-00251606}

Submitted on 1 Jan 1993

HAL is a multi-disciplinary open access archive for the deposit and dissemination of scientific research documents, whether they are published or not. The documents may come from teaching and research institutions in France or abroad, or from public or private research centers.
L'archive ouverte pluridisciplinaire HAL, est destinée au dépôt et à la diffusion de documents scientifiques de niveau recherche, publiés ou non, émanant des établissements d'enseignement et de recherche français ou étrangers, des laboratoires publics ou privés. 


\title{
Optical properties of GaAs/AlGaAs quantum dots realized by implantation induced intermixing
}

\author{
F.E. PRINS, F. ADLER, G. LEHR, S.Yu. NIKITIN ${ }^{(1)}$, H. SCHWEIZER and G.W. SMITH* \\ 4. Physikalisches Institut, Universität Stuttgart, Pfaffenwaldring 57, 70550 Stuttgart 80, Germany \\ * DRA Malvern, St. Andrews Rd, Malvern, Worcs, WR14 3PS, G.B.
}

\begin{abstract}
GaAs/AlGaAs quantum dots with diameter down to $70 \mathrm{~nm}$ have been realized by implantation induced intermixing. Photoluminescence studies demonstrate a high optical quality. From the systematic small blue shift of the dot luminescence with dot diameter it is shown that the dots reveal a steep radial potential and that the blue shift is caused by quantization. The increase of relative quantum efficiency of the dots with decreasing diameter can be explained by a model which takes the graded shape of the potential and the carrier capture from the barrier into account. Time-resolved measurement yield short carrier lifetimes in the dots. With decreasing dot diameter an increasing decay time and a slowed carrier cooling is observed.
\end{abstract}

\section{INTRODUCTION}

After the theoretical predictions of improved laser performance for quantum wires and dots $[1,2]$, many technological efforts have been made to realize such structures. Although many results have been reported about the optical properties of quantum wires, hardly any results are known regarding quantum dots.

One method to realize low dimensional structures is the implantation induced intermixing technique [3]. This method is based on the reduced thermal stability of a quantum well (QW) structure after introducing lattice damage by ion implantation. During annealing, the implanted parts of a $\mathrm{QW}$ will be intermixed, causing an enlargement of the band gap, while undamaged regions remain the original band gap. After implantation and annealing of a masked QW sample, the band gap of the unmasked parts is enlarged, resulting in a potential barrier for electrons and holes. These structures are buried and therefore suitable for optical studies and applications.

In this paper we present quantum dots realized by intermixing with diameters in the region ( $<100 \mathrm{~nm})$ where quantization effects can be expected. From the dot luminescence emission we estimate the shape of the radial potential and model the carrier capture from the intermixed barriers into the quantum dots. Time resolved measurements show increasing lifetimes and a slowed carrier cooling with decreasing diameter.

\section{EXPERIMENTAL}

The processed sample was a surface near $4.8 \mathrm{~nm}$ wide $\mathrm{GaAs}$ quantum well in $\mathrm{Al}_{0.33} \mathrm{Ga}_{0.67} \mathrm{As}$ barriers grown by molecular beam epitaxy. The distance from the $\mathrm{QW}$ to the surface was 15 $\mathrm{nm}$, which is an important condition for the realization of homogeneous nanostructures [4]. On top of the sample $60 \mathrm{~nm}$ thick $\mathrm{Au} / \mathrm{SiO}_{2}$ implantation masks have been defined by high resolution electron beam lithography. After evaporation and a lift-off process, fields of implantation masks with width of 400 down to $70 \mathrm{~nm}$ (measured by scanning electron microscopy) were realized. The structures have been implanted with $8 \cdot 10^{13} \mathrm{~cm}^{-2}{ }^{69} \mathrm{Ga}^{+}$

(1) Permanent address: Minsk Radioengineering Institut, Microelectronics Department, 6 P. Brovki Str., 220600, Republic Byelarus (C.I.S.) 


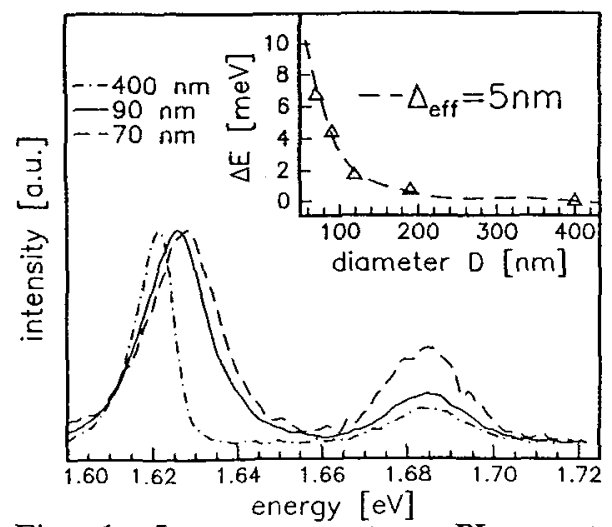

Fig. 1. Low temperature PL spectra of quantum dots with different diameter. The inset shows the observed blue shift in dependence of dot diameter, together with the calculated energy shifts in a radial potential $\mathrm{V}(\mathrm{r})=\mathrm{V}_{0}\left\{0.5+0.5 \operatorname{erf}\left\{\mathrm{r}-\mathrm{R} / 2 \Delta_{\text {eff }}\right\}\right)$.

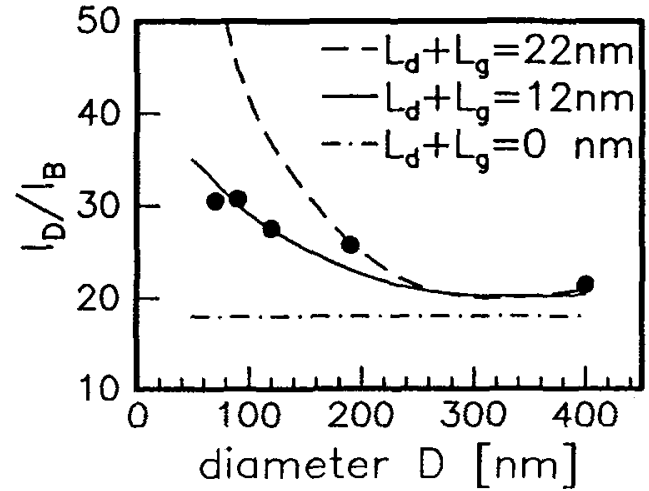

Fig. 2. Ratio of normalized dot and barrier intensities vs dot diameter. The curves represent calculations for different values of $L_{d}+L_{g}$.

and an energy of $40 \mathrm{keV}$. After removal of the masks in buffered HF, the intermixing of the implanted samples was performed in an annealing step, $925^{\circ} \mathrm{C} 60 \mathrm{~s}$.

For the optical studies, the samples were mounted in a He-bath cryostat. The samples have been excited by a Ti:Sapphire laser. Time resolved measurements have been carried out by exciting the dots with a synchronously mode-locked dye laser which yields pulses of $8 . .10$ ps. The emitted light was dispersed by two monochromators with subtractive assignment and detected by a fast microchannelplate photomultiplier with $\$ 20$ characteristics and a time resolution of about 30 ps.

\section{RESULTS}

In fig. 1 the low temperature time integrated photoluminescence (PL) spectra of quantum dots with different diameter are shown. From all fields with different dot diameter a high luminescence intensity is obtained. The spectra exhibit two clear emission lines, which can be attributed to the intermixed barriers (higher energies) and to the quantum dots (lower energies). The emission energy of the $400 \mathrm{~nm}$ wide dots is $1.62 \mathrm{eV}$, which is equal to the emission energy of the as grown sample, and shows, that no intermixing in the masked parts of the sample has occurred. This means that no ions penetrated through the implantation masks and damaged the quantum dots, as expected from the low implantation energy and the use of a surface near QW [4].

With decreasing dot diameter, an increasing shift to higher energies of the dot emission energy can be observed in fig. 1, while the barrier emission remains at constant energy. For dot diameters $>150 \mathrm{~nm}$ the blue shift is small, but for structures with diameter below $100 \mathrm{~nm}$ the shift is significant, up to $7 \mathrm{meV}$ for the $70 \mathrm{~nm}$ quantum dots, as is shown in the inset of figure 1. From the studies of quantum wires realized by this method, it is known, that the blue shift is partly caused by lateral defect diffusion, ion straggling during implantation and by quantization effects. The general accepted model for quantum wires is an errorfunction like lateral potential [3].

For the interpretation of the blue shift of the dot luminescence we assumed an errorfunction like radial potential with the parameter $\Delta_{\text {eff }}$ describing the steepness of the potential. The energy levels in the quantum dots with this potential shape have been calculated solving Schrödingers equation in cylindrical coordinates numerically using a matrix algorithm [5]. The results of the calculations for three different values of $\Delta_{\text {eff }}$ are also depicted in the inset of fig. 2. The best fit to the data is obtained for $\Delta_{\text {eff }}=5 \mathrm{~nm}$, which implies a very steep potential $[3,4,6,7]$. With this potential it is estimated that the observed blue shift is caused by radial quantization and that the contribution from the intermixing in the center of the dots is negligible. The value of $\Delta_{\text {eff }}=5 \mathrm{~nm}$ differs only little from the spread of the vacancy distribution under the masks due to the 
straggling of the ions, which has been estimated by Monte Carlo simulations [8] to be 3.5 $\mathrm{nm}$ in the depth of the QW.

The luminescence intensity of the quantum dots is remarkably high, especially compared to theoretical predictions [9]. In figure 2 we have depicted the ratio of the integrated PL intensity of the dot and the barrier emission $I_{D} / I_{B}$ in dependence of the dot diameter. The intensities $I_{D}$ and $I_{B}$ represent normalized values with respect to their filling factors. With decreasing dot diameter an increase of the ratio of intensities is observed, which is small compared to former result for quantum wires [6]. The increase of the relative quantum efficiency of the dot structures can be attributed to capture from carriers from the graded radial barrier into the dots.

The capture from carriers in quantum wires has been described by a simple model [6]. For the case of a cylindrical quantum dot we have made a similar calculation, which takes into account the effective diameter $D^{\prime}=D+2 L_{g}$ of the quantum dot, broadened by the graded potential, and a diffusion length $\mathrm{L}_{\mathrm{D}}$ of the carriers in the radial barrier. The different generation rates and internal quantum efficiencies in the dot and barrier regions are estimated by the ratio $R$ of the intensities of large unimplanted and implanted regions $\mathrm{R}=\mathrm{I}_{\text {unimpl }} / \mathrm{I}_{\text {impl }}$, 18 in our case. With this model we can calculate the relative quantum efficiency for different values of $L_{G}$ and $L_{D}$ and it shows that $I_{D} / I_{B}$ is mainly determined by the sum $L_{G}+L_{B}$. In figure 2 the results of calculations for 3 values $L_{G}+L_{B}$ are represented by the curves. The best fit to the data is obtained for $\mathrm{L}_{\mathrm{G}}+\mathrm{L}_{\mathrm{B}}=12 \mathrm{~nm}$. From the shape of our errorfunction like potential with $\Delta_{\text {eff }}=5 \mathrm{~nm}$ we obtain a value of $15 \mathrm{~nm}$ for the extend of the graded zone. Therefore we conclude that the increase of the relative quantum efficiency is only due to the graded potential and the high luminescence intensity cannot be explained by a strong carrier capture from the radial barrier. The lack of carrier diffusion and the high ratio of intensities of 18 for wide implanted an unimplanted regions can be explained by residual implantation damage. This is in good agreement with the small carrier lifetime of $70 \mathrm{ps}$ in the radial barriers, which allows hardly any diffusion in the radial barrier.

To investigate the carrier dynamics the lifetimes have been measured at different spectral position relative to the PL maximum (from $-6 \mathrm{~nm}$ to $+6 \mathrm{~nm}$ ). This allows the study of the carrier behaviour at the high and the low energy side of the PL emission. In the case of non resonant excitation (above the lateral barrier, $710 \mathrm{~nm}$ ) we also obtain the barrier lifetime. All lifetimes of the sample are relatively short, also in the case of the as grown sample $(170 \mathrm{ps})$, which is attributed to the vicinity of the surface. The radial barrier lifetime is $70 \mathrm{ps}$, independent of dot diameter indicating homogeneous implantation conditions for the barrier. This small value gives evidence of an efficient carrier

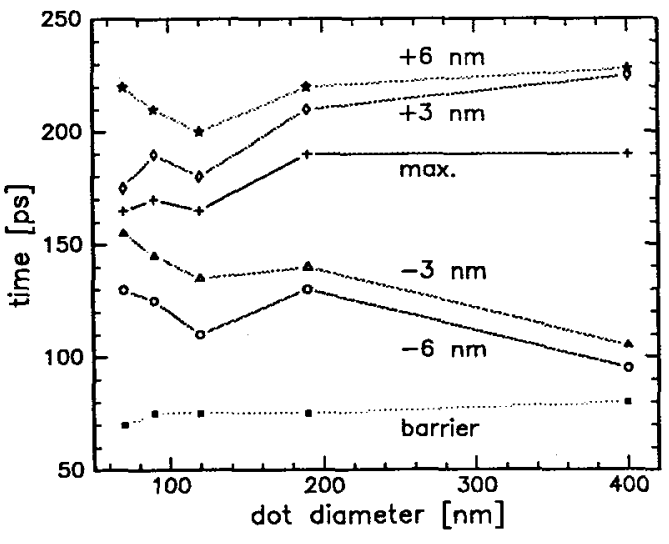

Fig. 3. Decay time of the PL vs dot diameter at different positions of the the PL-peak.

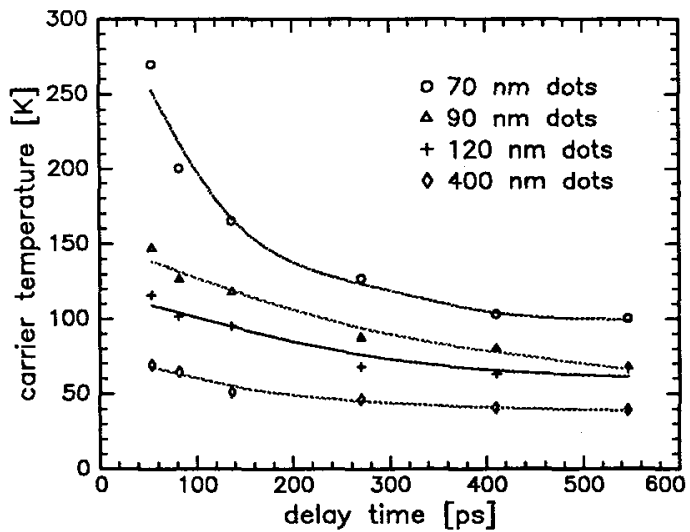

Fig. 4. Carrier temperature at various delay times after pulse excitation and different dot diameters. The spectra have been taken at $2 \mathrm{~K}$ and an excitation power of $106 \mathrm{kWcm}^{-2}$. 
loss process, which may be a strong nonradiative recombination due to residual implantation damage in the barrier regions.

Fig. 3 depicts the lifetimes in dependence of dot diameter at different spectral positions. At the high energy side the lifetimes increase with decreasing diameter, which could be a hint for a slower carrier relaxation. At the low energy side a decrease in the lifetimes is observed for smaller dots. We contribute this to a stronger nonradiative recombination in smaller dots, due to the higher defect density, caused by the lateral ion straggling. After resonant excitation $\left(\lambda_{\text {exc }}=742 \mathrm{~nm}\right)$ the carriers have less excess energy and energy relaxation is less important. Lifetime measurements under these conditions show the same results. Therefore we conclude, that the relaxation times are essentially shorter than the main recombination time (radiative or nonradiative).

A more detailed study of the carrier relaxation results in transient spectra at various time delays after the laser pulse (time window $54 \mathrm{ps}$ ). This allows a first rough determination of the carrier temperature in dependence of delay time (fig. 4), by fitting a straight line in the high energy side of the logarithmic spectra. In wide dots, strongly cooled carriers are observed even for short delay times, whereas carriers in small dots remain hot for a long delay time. Thus, a clear reduction of the carrier relaxation with decreasing dot diameter is concluded. The broadening of the PL of the smallest quantum dots also causes an increase of the estimated carrier temperature. This explains the different carrier temperatures at long delay times.

In analogy with similar experiments on quantum wires [10] we explain the reduced relaxation with the changed selection rules of momentum and energy conservation in low dimensions as predicted [9]. Broadening effects, which partly weaken the energy and momentum selection rules, must be assumed to explain the short lifetimes, even for small dots. The high carrier temperature may be due to an efficient Auger process, generating hot carrier which relax again.

\section{SUMMARY}

We have realized high quality quantum dots with diameter down to $70 \mathrm{~nm}$ and high luminescence intensity. With increasing dot diameter an increasing blue shift of up to $7 \mathrm{meV}$ for the smallest structures is observed. Assuming an errorfunction like radial potential we estimate that the shift is due to quantization in the confining potential. For the quantum dots we observe a small increase of the quantum efficiency with decreasing diameter, which is attributed to the graded shape of the potential. Lifetime measurements on the high energy side of the dot emission show an increasing decay times for the smallest dots. This is consistent with the observed slowed cooling of the carriers with decreasing diameter.

\section{ACKNOWLEDGEMENT}

The authors would like to thank $H$. Gräbeldinger and $P$. Bloching for their technical assistance and M.H. Pilkuhn for stimulating discussions. This work has been supported by ESPRIT BRA Contract No. 3043 LATMIC.

\section{REFERENCES}

[1] Y. Arakawa and H. Sakaki, Appl. Phys. Lett. 40, 939 (1982)

[2] M. Asada, Y. Miyamoto, Y. Suematsu, Jpn. J. Appl. Phys. 24, L95 (1985)

[3] J. Cibert, P.M. Petroff, G.J. Dolan, S.J. Pearton, and J.H. English, Appl. Phys. Lett. 49, 1275 (1986)

[4] F.E. Prins, G. Lehr, M. Burkhard, H. Schweizer, M.H. Pilkuhn, G.W. Smith, Appl. Phys. Lett. 62 (12), 1365 (1993)

[5] F.E. Prins, G. Lehr, S.Yu. Nikitin, H. Schweizer, G.W. Smith, to be published in Phys. Rev. B

[6] H. Leier, A. Forchel, B.E. Maile, G. Mayer, J. Hommel, G. Weimann, W. Schlapp, Appl. Phys. Lett. 56, 48 (1990)

[7] C. Vieu, M. Schneider, G. Benassayag, R. Planel, L. Birotheau, J. Y. Marzin, B. Descouts, J. Appl. Phys. 71, 5012 (1992)

[8] J.F. Ziegler, J.P. Biersack, U. Littmark, The Stopping of Ions in Solids (Pergamon, New York, 1985)

[9] H. Benisty, C.M. Sotomayor-Torrès, C. Weisbuch, Phys. Rev. B 44, 10945 (1991)

[10] G. Mayer, F.E. Prins, G. Lehr, H. Schweizer, H. Leier, B.E. Maile, J. Straka, A. Forchel, G. Weimann, Phys. Rev. B 47, 4060 (1993) 